\title{
Historisch-biographische Anmerkungen zu den vier vom Historischen Verein für Württembergisch Franken mit Unterstützung der Sparkasse Schwäbisch Hall-Crailsheim erworbenen Brenz-Drucken (Mai 2013)
}

Allgemeines (für alle vier Drucke):

Verfasser: Johannes Brenz (1499-1570), Prediger und Reformator von Schwäbisch Hall 1522-1548, Flucht und Exil 1548-1552, Landespropst und Reformator für Württemberg in Stuttgart 1553-1570.

Vorbesitzer: Bibliothek des Sammlers Martin Schupp; geb. am 19.07.1936 in Tübingen, gest. ebd. am 28.08.2011 (Grab auf dem Stadtfriedhof Tübingen); Schulen in Tübingen (zuletzt Uhland-Gymnasium), 1954-58 Evang.-theol. Seminare Schöntal und Urach, seit 1958 Theologiestudium im Tübinger Stift (dazwischen Erlangen), 1965 Erstes theol. Examen; (Pfarr)vikariate bis 1979, 1979-1989 Pfarrer in Baumerlenbach bei Öhringen, dann Altenheim-Seelsorger in Tübingen bis 2009; Bibliothek und Sammlung Schupp aufgelöst 2012/13 durch mehrere Händler (ohne Dokumentation).

Erwerbung der vier Drucke von: Peter Kiefer, Buch- und Kunstauktionen, 75172 Pforzheim, Steubenstraße 36, Auktion 84 am Freitag, 19. April 2013.

\section{1) Brenz, Johannes: Ein Sermon zu allen Christen von der Kirche, [Erfurt: Wolfgang Stürmer], 1523}

Titel: Eynn Sermon II tzu allen Christen / von II der Kirche, vn̄ von jrem schlus= || sel vnnd gewalt / auch vonn II dem ampt der priester. I| Geprediget durch Johan = I| nem Brentz / Anno. M. D. xxiij. || [TE] ॥

Druck: [Erfurt: Wolfgang Stürmer], 1523

4 Bl. in Quartformat, letzte S. leer, mit Holzschnitt-Titeleinfassung.

Bibliographische Nachweise: Verzeichnis der Drucke des 16. Jhs. (VD 16): B 7876; Köhler, Bibliographia Brentiana 1904 (Repr. 1963) Nr. 5; Weller, Repertorium typographicum 1864 Nr. 2369; Martin v. Hase, Bibliographie der Erfurter Drucke von 1501-1550, 3. Aufl. 1968 S. 17 Nr. 126 und S. 169 (Stürmer TE 2); Johannes Luther, Titeleinfassungen der Reformationszeit, 1973, S. XVIII und Tf. 60; Index Aureliensis Nr. 124.293; Buchauktionshaus Kiefer, Pforzheim, Katalog 84, 2013 Nr. 65.

Edition: Johannes Brenz, Frühschriften 1, Tübingen 1970 S. 15-22 (S. 16: Druck B).

Literatur: Hartmann/Jäger, Brenz, Bd. 1, 1840 S. 55-58; Brecht, Chronologie von Brenzens Schriftauslegungen und Predigten, BWKG 64, 1964 S. 64; Brecht, Frühe Theologie 1966 S. 25-36. Zum Drucker s. Reske, Die Buchdrucker des 16. und 17. Jhs., 2007 S. 201f.). 


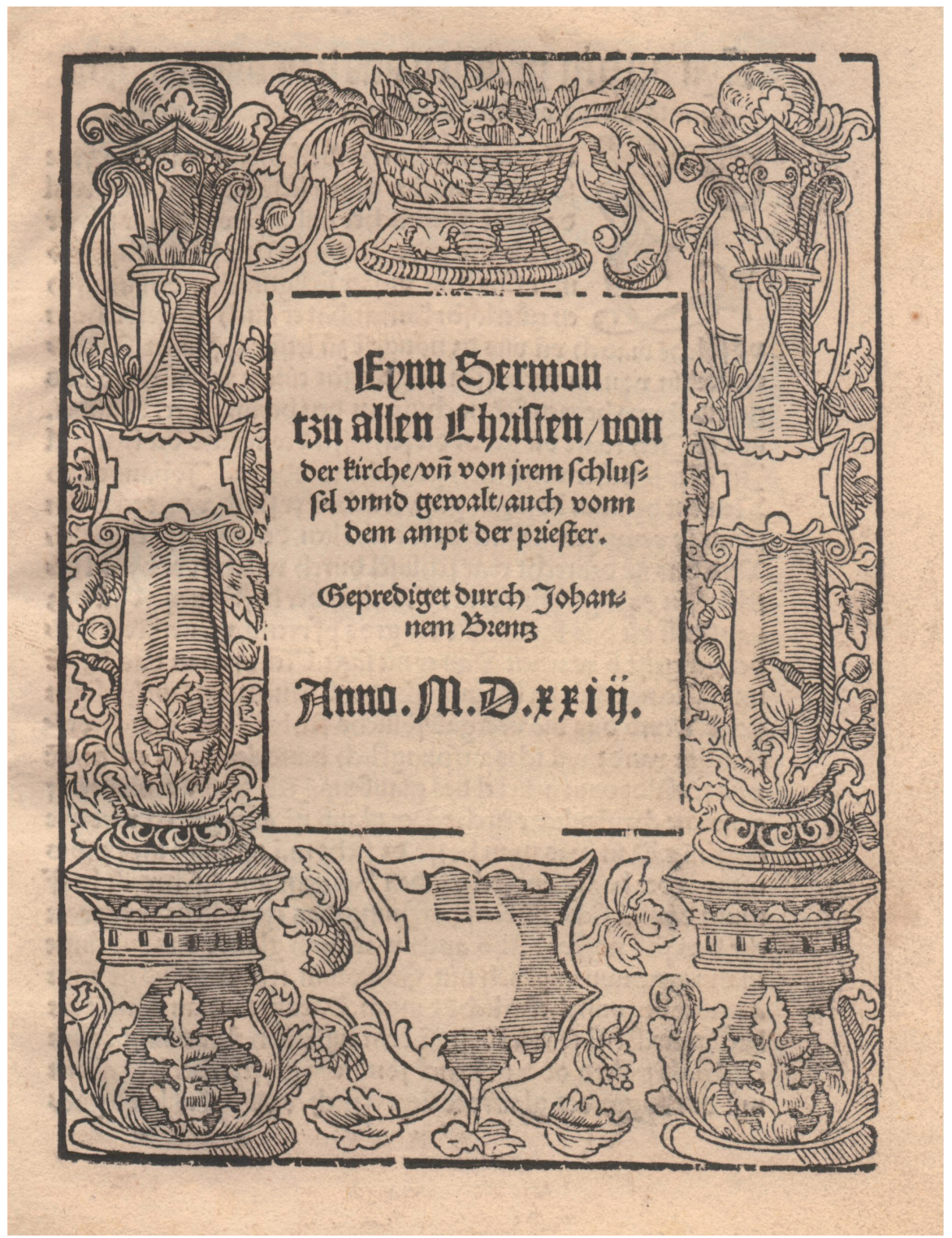

Eynn Sermon tzu allen Christen von der Kirche, ṽ̃ von jrem schlussel vnnd gewalt auch vonn dem ampt der priester. Geprediget durch Johannem Brentz. Anno. M. D. xxiij (Titelblatt der vielleicht ersten gedruckten Brenz-Schrift). 
Provenienz: Martin Schupp (Exlibris), s. oben.

Anmerkungen: Neben dem „Sermon von den Heiligen“ vom Jakobitag (25.5.) 1523 (das StA Hall besitzt davon einen frühen Augsburger Nachdruck: Köhler Nr. 1, VD 16: B 7872) gehört diese zweite frühe Brenz-Flugschrift, der „Sermon von der Kirche“, zu den beiden ersten selbstständig gedruckten Schriften des Haller Reformators. Die vorliegende Ausgabe ist ein Erfurter Nachdruck der bei Jörg Nadler in Augsburg im selben Jahr erschienenen Erstausgabe (Köhler Nr. 4, VD 16: B 7874), auf die noch weitere mindestens sechs Nachdrucke folgten. Der Sermon wendet sich wie derjenige von den Heiligen klar und programmatisch gegen die römische Kirche und deren Amtsverständnis. Die Kirche als Versammlung der Glaubenden ist nach Brenz eine christologische Größe, eine im Grunde verborgene, geistliche Kirche unter dem Kreuz, in die die Glaubenden durch die Taufe in Christus „eingeleibt“ sind und in der sie durch den Glauben die Schlüsselgewalt der Vergebung ausüben. Da dem „Sermon von der Kirche“ das Evangelium des Sonntags Quasimodogeniti (Joh. 20) zugrunde liegt, könnte die Predigt an diesem Sonntag, der im Jahr 1523 auf den 12. April fiel, gehalten worden sein. Sie wäre dann der ältere von den beiden Sermonen von 1523 und damit die erste gedruckte Brenz-Schrift.

\section{2) Brenz, Johannes: Die rechte und wahrhaftige Auslegung des ganzen Ge- setz Gottes, [Frankfurt a. M.: Peter Braubach], 1544 / Hagenau: Valentin Kobian, 1539}

Titel: Die rechte vnd || warhafftige $A u ß=\|$ legung des gantzen Gesatz || Gottes / allen menschen || hoch von nôtten zu || wissen. || Durch || Ioannem Brentium. || Anno M. D. xliiij. || [TE] ॥

[Am Ende, Bl. 202a:] Getrůckt Zů Hagenaw / bei Veltin Kobian. alß II mann zalt Tausent Fünffhundert Dreissig I| Neün Jar. II [Holzschnitt-Leiste] || Druck des Titelbogens (Bog. *): [Frankfurt a.M.: Peter Braubach], 1544; Druck der übrigen Bogen (A-Ddd): Hagenau: Valentin Kobian, 1539.

206 Bl. in Quartformat, Titelrücks., Bl. *4b und letzte S. leer; gedruckte Marginalien, Kolumnentitel; mit Holzschnitt-Titeleinfassung (von Hans Brosamer) und 5 Hschn-Initialen; B1. *2a-*3a Vorrede, undat. (von Brenz?), *3b-*4a Inhaltsverzeichnis.

Übersetzer: Wolfgang Maler (um 1490-1552), Archidiaconus an St. Michael 1530-1549 (Pfarrerbuch Württ. Franken 2, 1981 S. 284 Nr. 1664).

Bibliogr. Nachweise: VD 16: B 7743 (vgl. ZV 2439, wohl fehlerhaft); Köhler, Bibliographia Brentiana Nr. 137; Index Aureliensis Nr. 124.417; Antiquariat Edelmann Nürnberg, Katalog 75, 1972 (Reformationsdrucke) von W. Gose, S. 14 Nr. 29 (das vorliegende Exemplar!); Herwarth v. Schade, Peter Braubach in Frankfurt (1540-1567). Ein Werkverzeichnis. In: Archiv f. Geschichte d. Buchwesens 21, 1980 Sp. 870 Nr. 40; Katalog Kiefer 84, 2013 Nr. 58 (mit Abb., ebenfalls dieses Exemplar). 
Literatur: Brecht, Chronologie von Brenzens Schriftauslegungen und Predigten, BWKG 64, 1964 S. 62f. Bodo Gotzkowsky, Die Buchholzschnitte Hans Brosamers. Ein bibliogr. Verzeichnis ihrer Verwendungen, 2009 S. 348 Nr. 1 und 349f. (Abb. TE). Zu den beiden Druckern s. Reske, Die Buchdrucker des 16. und 17. Jhs., 2007 S. 322 (Kobian), 227 f. (Braubach in Frankfurt) und 830f. (Braubach in Hall).

Provenienz: Martin Schupp (Exlibris), s. oben.

Anmerkungen: Diese 21 Bußpredigten über Bibeltexte aus Lukas 6 (und Matthäus 5) sind erstmalig 1539 in Hagenau bei dem Drucker Kobian erschienen unter dem Titel „Das Sechst Capitell des Euangelisten Luce, sampt dem Fünfften Mathei, von der rechten warhafftigen außlegung des Gesetzes, inn 21 predigt verfasset" (Köhler Nr. 107; VD 16: B 7744). Wie es hier in der Widmungsvorrede Malers an den Haller Rat heißt, sind diese Predigten „hie zu Hall vor etlicher zeyt durch vnsern lieben Herren prediger Johann Brentzen geprediget / vnd zu latein im druck auß gangen“ (B1. 2a); sie wurden dann durch den Brenz-Kollegen Maler übersetzt und 1539 von Kobian, der viel für Brenz gedruckt hat, veröffentlicht. Ob es sich bei der genannten lateinischen Vorlage um entsprechende Abschnitte aus Brenzens Lukas-Homilien von 1537 handelt (Köhler Nr. 93, VD 16: B 7728; vgl. unten Nr. 8), müsste noch untersucht werden. Die vorliegende Neuauflage (sog. Titelauflage) des Drucks von 1539 wurde im Jahr 1544 von dem großen Frankfurter Druckerverleger und Freund von Brenz Peter Braubach (um 1500-1567) herausgegeben. Dieser hatte zuerst ebenfalls in Hagenau gedruckt und war danach von Juli 1536 bis 1540 (und nochmals 1544/45 von Frankfurt aus) der Erstdrucker von Hall. Dabei hat Braubach den noch vorhandenen Satz des 1543 in Hagenau verstorbenen Druckers Kobian übernommen und ihn nur mit einem neuen Titelbogen (4 Blatt) versehen. Dieser bietet einen etwas kürzeren Titel samt neuem Druckjahr 1544, dazu ein undatiertes, wohl von Brenz verfasstes neues Vorwort und ein gegenüber 1539 neues Inhaltsverzeichnis. Da die Titeleinfassung das Frankfurter Wappen zeigt, dürfte der Titelbogen auch tatsächlich dort gedruckt worden sein, obwohl ihn möglicherweise auch Braubachs Schwager, der 1543 gestorbene Drucker Pankratius Queck in Hall, von dem nur firmierte Drucke von 1543 nachgewiesen sind, noch hergestellt haben könnte.

Das Buch bietet einen umfassenden Überblick über die wichtigsten Themen der städtischen Reformations- und Sittenordnung: Sonntagsheiligung, Apostelfeste, Armenfürsorge, Totschlagsdelikte, Eheordnung, Eidschwören, Rache, Nächstenliebe, Diebstahl, Unterschied von Gesetz und Evangelium, Kaufen und Verkaufen, Zinsnehmen, üble Nachrede, falsche Lehre und Anderes. 


\section{3) Brenz, Johannes: Von rechter wahrer christlicher Buß, Schwäbisch Hall: Peter Frentz, 1546}

Titel: Von Rechter || warer Christlicher Bůß / v̄̄ || notwendigen dingen darzů gehơrig / II Auch den Christlichen Sacra= II menten vnd etlichen kirchen II gebreüchen / Fünff vnd || zweintzig Pre= || digt / || Herrn Johan Brentzen. || Gedruckt zů Schwebischen || Hall durch Peter Frentzen / II im Jar / II [Querstrich] || M. D. XLVI. ॥

[Am Ende, Bl. G7b:] Gedruckt zů Schwåbischen II Hall / durch Peter Fren= II tzen / im Jar. || [Querstrich] || M.D.XLVI. I|

Druck: Schwäbisch Hall: Peter Frentz, 1546

248 Bl. in Oktavformat, ab Bl. 10 foliiert mit ii-ccxxviii (mehrfach fehlerhaft!), dann 13 Bl unfoliiert; Titelrücks., Bl. +8, Bl. G7a und letztes Bl. leer; gedruckte Marginalien, Kolumnentitel; Bl +2a $-+4 \mathrm{~b}$ Vorrede, undat. (von W. Maler?), Bl. $+5 \mathrm{a}-+7 \mathrm{~b}$ drei Gebete); Bl. F3a-F5a Druckfehlerverzeichnis, F5b-G6b Register.

Bibliographische Nachweise: VD 16: B 7848; Köhler, Bibliographia Brentiana Nr. 149; German (Schwäbisch Hall) 1916 S. 103 Nr. 7; Pegg (Copenhagen) 432; Katalog Kiefer 84, 2013 Nr. 77.

Literatur: Hartmann/Jäger, Brenz, Bd. 2, 1842 S. 470 (diese Ausgabe?); Brecht, Chronologie von Brenzens Schriftauslegungen und Predigten, BWKG 64, 1964 S. 63 und 72. Zum Drucker s. Reske, Die Buchdrucker des 16. und 17. Jhs., 2007 S. 831.

Provenienz: a) Schweinslederband mit Blindpressung: Monogramm „K·R“ und Datierung „1554“, Rollenstempel mit bibl. Motiven; b) hdschr. Einträge (Bibelworte, Gebete etc.), dabei Vermerk: „Wolf Niderleuttner: Geschriben des 1625. Jhars Den 28 dag Maÿ“; c) „Liber Ecclesie B.M.V. [Ort ausradiert] 1670“; d) Martin Schupp (Exlibris), s. oben.

Anmerkungen: Brenzens 25 Bußpredigten sind, obwohl natürlich deutsch gehalten, zuerst im August 1544 lateinisch unter dem Titel „De poenitentia et iis, quae ad poenitentiam agendam necessaria sunt, homiliae viginti quinque" bei Braubach in Hall gedruckt erschienen (Köhler Nr. 127, VD 16: B 7844); weitere Ausgaben dieser lateinischen Fassung wurden 1545, 1550 und 1557 gedruckt (Köhler 141, 179 und 331) und als Teil von Brenzens Postille von 1556 (Köhler 297); sie wurden dabei z. T. mit weiteren Brenztexten bzw. mit Beigaben anderer Verfasser, z. B. Melanchthons, verbunden. Herausgeber und Bearbeiter (!) der Homiliae ist der oben genannte Wolfgang Maler (s. dessen Widmungsvorrede an Johann Hess in Breslau, dat. Hall 1. September 1544). Die hier vorliegende deutsche Übersetzung bzw. eigentlich Rückübersetzung (einzige Ausgabe; neben ihr gibt es nur noch eine Übersetzung ins Dänische, Kopenhagen 1563) nennt keinen Übersetzer und auch das Vorwort ist weder datiert noch unterschrieben. Man darf jedoch annehmen, dass auch hier Wolfgang Maler sowohl der Übersetzer als auch der Herausgeber und Vorwort-Verfasser ist. Brenz selbst kommt für die im Übrigen recht gute Übersetzung wohl kaum infrage. 


\section{4) Brenz, Johannes: Meinung von Mitteldingen oder Adiaphoris. [Magdeburg: Michael Lotter, 1549/50]}

Titel: Des Herrn Johan= $\|$ nis Brentij meinung von Mittel= $\|$ dingen oder Adiaphoris / || sehr nützlich zu || lesen. || II. Corinth: VI. || Ziehet nicht am frembden Joch mit den vngleubi= $\|$ gen / Denn was hat die gerechtigkeit für genies mit der $\mathrm{vn}=\|$ gerechtigkeit? Was hat das Liecht fur gemeinschafft mit $\|$ dem Finsternis? [...] vnd ir sôllet mei= ॥ ne So̊ne vnd Töchter sein / spricht der II Allmechtiger Herr. II

Druck: [Magdeburg: Michael Lotter, 1549/50]

4 B1. in Quartformat, unpaginiert, Bogensignatur: A.

Bibliographische Nachweise: VD 16: B 7789; Köhler, Bibliographia Brentiana Nr. 676; Katalog Bibliothek Knaake (Reformationsschriften) 1908, Bd. III Nr. 114; Pegg (Copenhagen) 421; Antiquariat Kocher-Benzing Stuttgart, Katalog 105, 1979 Nr. 18; Katalog Kiefer 84, 2013 Nr. 56.

Internet: S. das Forschungsprojekt „Controversia et Confessio. Quellenedition zur Bekenntnisbildung und Konfessionalisierung (1548-1580)“ der Mainzer Akademie der Wissenschaften (Prof. Dr. Irene Dingel) und dessen Homepage (mit Information zur Brenz-Schrift und Textwiedergabe unter „Datenbank“).

Literatur: Hartmann/Jäger, Brenz, Bd. 2, 1842 S. 379 f. und 398-403, vgl. auch Bd. 1, 1840 S. $189-$ 191; I. Dingel (Hg.): Der Adiaphoristische Streit (1548-1560), Bd. 2 in der Reihe „Controversia und Confessio“, Göttingen 2012. Zum Drucker s. Reske, Die Buchdrucker des 16. und 17. Jhs., 2007 S. 580).

Provenienz: a) H. G. Corsepius P[farrer?], 1935; b) Martin Schupp (Exlibris), s. oben.

Anmerkungen: Die Flugschrift aus der Interimszeit (1548/52), die wohl kaum auf Initiative von Brenz erschien, aber wahrscheinlich doch mit dessen Wissen, druckt offenbar eine Passage aus einer noch nicht identifizierten Brenz-Schrift $\mathrm{ab}$ und gehört thematisch in den Zusammenhang des sog. Adiaphoristischen Streits. In dieser politisch und kirchenpolitisch folgenreichen, aber im Grunde rein theologischen Auseinandersetzung unter den Nachfolgern Luthers ging es um die Frage, wie heilsnotwendig oder unschädlich für die ,wahre Kirche“ die Adiaphora, d.h. ,freie Mitteldinge“ wie kirchliche Riten und Bräuche sind. Melanchthon und die „Philippisten“ vertraten dabei eine freiere, positive Haltung gegenüber den Adiaphora, während die strengen Lutheranhänger (die „Gnesiolutheraner“, wie z. B. Flacius IIlyricus), hier jeden kaiser- und katholikenfreundlichen Kompromiss ablehnten. Brenz zeigt in dieser Kontroverse eine vermittelnde Haltung, aber mit klarer theologischer Position, wie sie bei ihm schon in den frühen Jahren der Reformation zu finden ist. 


\section{Anhang: \\ Brenz-Drucke aus der Sammlung Schupp, die im Februar und April 2013 (Kiefer-Auktionen 83 und 84) vom Stadtarchiv Schwäbisch Hall erworben wurden}

1) Brenz, Johannes: Apologiae Confessionis Illustriss. Principis ac Domini, D. Christophori Ducis Vuirtenbergen. etc. [EA des 2. Teils der Apologie zur Confessio Virtembergica, Pericopae II/1]

Frankfurt a.M.: Peter Braubach, 1557 (180 Bl. in $\left.4^{\circ}\right)$

Köhler 329 (EA Teile 1-4: 288, 308, 330, 355); Teil von VD 16: B 7486 (= Pericopae II und III); v. Schade (Braubach) 222; Antiquariat Kiefer, Pforzheim, Auktionskatalog 84, 2013 S. 7 Nr. 45.

2) Brenz, Johannes: Ecclasiastes Solomonis, cum commentariis, iuxta piis atque eruditis Iohannis Brentii, per Hiobem Gast e Germano in Latinum tralatus [TE] [lat. EA des Prediger-Kommentars; mit Widmungsvorrede Gasts an Lgf. Philipp v. Hessen]

Hagenau: Johann Setzer, 1528 (304 Bl. in $8^{\circ}$ )

Köhler 28; VD 16: B 3643; Benzing (Haguenau) S. 79 Nr. 79; Index Aurel. 124.317; Katalog Kiefer 84, 2013 S. 8 Nr. 59.

3) Brenz, Johannes: Enarrationvm Evangeliorvm Dominicalivm. Pars Prima. Cum praefatione Ioannis Pollicarii [EA von Bd. 1 der Pollicarius-Postille, Widmungsvorrede von Johannes Pollicarius (1524-1586?) in Weißenfels an Hz. August v. Sachsen]

Erfurt: Wolfgang und Gervasius Stürmer, 1550 (404 Bl. in $8^{\circ}$ )

Köhler 184 (EA Bd. 2: 185); VD 16: B 7591 (I und II); v. Hase (Erfurt) S. 159f. Nr. 1070; Index Aurel. 124.461; Brecht in BWKG 64, 1964 S. 62; Katalog Kiefer 84, 2013 S. 8 Nr. 61 (mit Abb.).

4) Brenz, Johannes: Explicatio Epistolae Pauli ad Galatas [TE] [3., revidierte Ausg. des Galater-Kommentars von 1546; wichtige Vorrede von Brenz an Johann Hess (1490-1547) in Breslau] Frankfurt a.M.: Peter Braubach, 1548 (336 Bl. in $8^{\circ}$, am Schluss DrM)

Köhler 161 (EA: 152); VD 16: B 7609; v. Schade (Braubach) 97; Pegg (Belg./ Neth.) 331; Pegg (Swed.) 147; Bodo Gotzkowsky, Die Buchholzschnitte Hans Brosamers. Ein bibliogr. Verzeichnis ihrer Verwendungen, 2009 S. 340 und 343 (zur DrM); Brecht in BWKG 64, 1964 S. 58 und 60, BWKG 70, 1970 S. 7 und in BWKG 100, 2000 S. 236; Antiquariat Edelmann Nürnberg, Katalog 75, 1972 
(Reformationsdrucke, von W. Gose), S. 14f. Nr. 30 (dieses Exemplar); Katalog Kiefer 84, 2013 S. 8 Nr. 62 (mit Abb.).

Angebunden: Brenz: Philipper-Kommentar, 1548 (s. unten).

5) Brenz, Johannes: Explicatio Epistolae Pauli Apostoli ad Philippenses [TE] [2. Ausg. des Philipper-Kommentars von 1548, hrsg. von Michael Gräter (14921562) in Schwäb. Hall]

Frankfurt a.M.: Peter Braubach, 1548 (152 Bl. in $8^{\circ}$ )

Köhler 159 (EA: 158); VD 16: B 7615; v. Schade (Braubach) 96; Index Aurel. 124.440; Pegg (Strasbourg) 419; Pegg (Belg./Neth.) 330; Hartmann/Jäger II,1842 S. 166 und 471; Brecht in BWKG 64, 1964 S. 58 und 60 und BWKG 70, 1970 S. 7; Bodo Gotzkowsky, Die Buchholzschnitte Hans Brosamers. Ein bibliogr. Verzeichnis ihrer Verwendungen, 2009 S. 340 und 342 f. (zur DrM); Antiquariat Edelmann Nürnberg, Katalog 75, 1972 (Reformationsdrucke, von W. Gose), S. 14 f. Nr.30 (dieses Exemplar); Katalog Kiefer 84, 2013 S. 8 Nr. 62. Vorgebunden: Brenz: Galater-Kommentar, 1548 (s. oben).

6) Brenz, Johannes: In D. Iohannis Evangelion Exegesis, per authorem iam nouißime diligenter reuisa, ac multis in locis locupletata [TE] [5. Ausg., revid. Fassung des Johanneskommentars von 1527/28, mit Vorrede an die Pfarrer im Kraichgau]

Hagenau: Johann Setzer, September 1532 (360 Bl. in $8^{\circ}$ )

Köhler 57 (EA: 22); VD 16: B 7710; Benzing (Haguenau) S. 98 Nr. 1; Index Aurel. 124.348; Hartmann/Jäger I, 1840 S. 169; Brecht in BWKG 64, 1964 S. 55 und 60; Katalog Kiefer 84, 2013 S. 9 Nr. 68.

Vorgebunden: Agricola, Johann: In Lucae Evangelium, Adnotationes, Hagenau: Joh. Setzer, 1529 (VD 16: A 1003).

Angebunden: Brenz: Epistola de verbis Domini, 1526 (s. unten).

7) Brenz, Johannes: Epistola Ioannis Brentii de uerbis Domini, Hoc est corpus meum, opinionem quorundam de Eucharistia refellens. [EA des Briefs an Martin Bucer (1491-1551) vom 3.10.1525]

[Hagenau: Johann Setzer, 1528] (8 B1. in $8^{\circ}$, 1 Hschn-Initiale B1. A2a)

Köhler 671 (vgl. Nr. 305 und S. 332); VD 16: B 7597; Benzing (Haguenau) S. 71 Nr. 34; Index Aurel. 124.303; Katalog Kiefer 84, 2013 S. 9 Nr. 68 (mit Abb.). Vorgebunden: a) Agricola, Johann: In Lucae Evangelium, Adnotationes, Hagenau. Joh. Setzer, 1529 (VD 16: A 1003); b) Brenz: In D. Johannis Evangelion Exegesis, 1528 (s. oben).

8) Brenz, Johannes: In Evangelii quod inscribitvr, secundvm Lvcam, duodecim posteriora Capita, Homiliae octoginta [TE] [2. Ausg. des 2. Teils der Lukas- 
Homilien von 1540, EA von Teil 1: 1537; Widmungsvorrede an Kanzler Georg Vogler (1486/87-1550) in Ansbach]

Frankfurt a.M.: Peter Braubach, 1542 / [am Schluss:] 1543 (318 Bl. in 2ªm Schluss DrM)

Köhler 120 (EA von Teil 2: 108, von Teil 1: 93); VD 16: B 7731; v. Schade (Braubach) 25; Hartmann/Jäger II, 1842 S. 113-115; Brecht in BWKG 70, 1970 S. 6; Katalog Kiefer 84, 2013 S. 9 Nr. 69.

9) Brenz, Johannes: In Exodvm Mosi commentarius [EA des Exodus-Kommentars, Vorwort an Kanzler Nikolaus Müller gen. Mayer (um 1490-1549) in Stuttgart]

Schwäbisch Hall: Peter Braubach, 1538 / [am Schluss:] 1539 (360 Bl. in $8^{\circ}$ )

Köhler 102; VD 16: B 7745; German (Schwäb. Hall) S. 67 Nr. 23; Hartmann/ Jäger II, 1842 S. 65-67; Pressel, Anecdota Brentiana 1868 S. XVII Nr. 164; Brecht in BWKG 64, 1964 S. 58 und 60; Katalog Kiefer 84, 2013 S. 9 Nr. 70 (mit Abb.).

Angebunden: Artopoeus, Petrus: Evangelicae Conciones Dominicarvm tocius anni, Wittenberg: Georg Rhau, 1537 (VD 16: B 1375).

10) Brenz, Johannes: Sententia, De Libello D. Henrici Bvllingeri, cvi Titvlvs est. Tractatio verborvm Domini: In Domo Patris mei Mansiones mvltae svnt etc. [DrM] [lat. EA; abgefasst als Brief an Hz. Christoph v. Württ.]

Tübingen: Ulrich Morhart d. Ä. Erben, 1561 (36 Bl. in $4^{\circ}$ )

Köhler 387; VD 16: B 7868; Index Aurel. 124.317; Hartmann/Jäger II, 1842 S. 386; E. Herkenrath: Bullinger-Bibliographie Bd. 2, 1977 S. 8 Nr. 1022; Kata$\log$ Kiefer 84, 2013 S. 9 Nr. 72.

11) Brenz, Johannes: Syntagma eorvm, qvae nomine illvstrissimi Principis ac Domini, D. Christophori Ducis Virtembergensis (...) in Synodo Tridentina Per Legatos eius, acta sunt [3. erweiterte Ausg. des latein. Berichts vom Besuch der Württemberger auf dem Trienter Konzil] [Schwäbisch Hall: Peter Frentz], 1553 (76 Bl. in $8^{\circ}$ )

Köhler 244 (EA: 242); VD 16: B 7896; Index Aurel. 124.508; Hartmann/Jäger II, 1842 S. 215; Schnurrer, Erläuterungen 1798 S. 214; Benzing (Strasbourg) 480; Katalog Kiefer 84, 2013 S. 9 Nr. 73.

12) Brenz, Johannes: Von der Mayestet Vnsers lieben Herrn vnd einigen Heilands Jesu Christi / zů der gerechten Gottes / auch von der waren gegenwürtigkeit des Leibs vnnd Blůts Christi im Nachtmal [2. Ausg. der dt. Fassung der Schrift gegen die Zwinglianer Petrus Martyr und Heinrich Bullinger von 1562] Tübingen: Ulrich Morhart d. Ä. Witwe, 1562 (36 Bl. in $4^{\circ}$ ) 
Köhler 407 (dt. EA; 406, lat. EA 403); VD 16: B 7788; Hartmann/Jäger II, 1842 S. 386-388; Katalog Kiefer 84, 2013 S. 9 f. Nr. 75.

13) Brenz, Johannes: Das Bůch Richter vnnd Ruth / außgelegt durch Johann Brentzen. Durch Hiob Gast verteütschet. M.D.XXXIX. [TE] Augsburg: Heinrich Steiner, August 1539 [DrM] (224 Bl. in 8)

Köhler 104 (dt. EA); VD 16: B 7764; Katalog Kiefer 84, 2013 S. 7, Nr. 52.

14) Brenz, Johannes: Acta apostolorvm. [TE] Das Buch der Apostel geschicht Die recht vnd Haubthistoria der ersten heyligen Christlichen Kirchen. Außgelegt durch Johannem Brentium / vnd in C.XXII. Predigten getheylet. Sampt einer Vorrede Brentij / von nütz vnd lobe dises Buchs / vor nie im Druck außgangen. Nürnberg M.D.LIIII.

Nürnberg: o. Dr. [wohl Joh. vom Berg und Ulrich Neuber, wie Köhler 206], 1554 $\left(414 \mathrm{Bl}\right.$. in $\left.2^{\circ}\right)$

Köhler 276 (2. dt. Ausg., EA lat.: 1535, Kö 75; EA dt.: 1551, Kö 206); VD 16: B 7694; Katalog Kiefer 83, 2013 S. 20f., Nr. 184.

Ausstellung der vom Stadtarchiv Hall 2012/13 erworbenen 14 Brenz-Drucke (Kundenhalle der Sparkasse Schwäbisch Hall - Crailsheim, Eröffnung 14. Juni 2013):

Folgende Drucke wurden erworben (Beschreibungen s. im Internet unter StA Hall, Neuerwerbungen; unterstrichen: oben nicht aufgeführte Drucke, also wohl nicht Provenienz Schupp):

Köhler 28, 57, 103 [tatsächlich: 102], 104, 120, 159/161, 184/185 (nicht Prov. Schupp), 244, 276 (Prov. Schupp; Kiefer 83), 329, 387, 407, 461 (nicht Prov. Schupp), 671. 